\title{
Supplying Conditions for Having up to 1000 Degrees of Freedom in the Onset of Inflation, Instead of 2 to 3 Degrees of Freedom, Today, in Space-Time
}

\author{
Andrew Walcott Beckwith \\ Physics Department, College of Physics, Chongqing University, Huxi Campus, Chongqing, China \\ Email: Rwill9955b@gmail.com, abeckwith@uh.edu
}

How to cite this paper: Beckwith, A.W. (2018) Supplying Conditions for Having up to 1000 Degrees of Freedom in the Onset of Inflation, Instead of 2 to 3 Degrees of Freedom, Today, in Space-Time. Journal of High Energy Physics, Gravitation and Cosmology, 4, 143-151.

https://doi.org/10.4236/jhepgc.2018.41013

Received: November 14, 2017

Accepted: January 28, 2018

Published: January 31, 2018

Copyright $\odot 2018$ by author and Scientific Research Publishing Inc. This work is licensed under the Creative Commons Attribution International License (CC BY 4.0).

http://creativecommons.org/licenses/by/4.0/

(c) (i) Open Access

\begin{abstract}
The following document attempts to answer the role additional degrees of freedom have as to initial inflationary cosmology, i.e. the idea is to cut down on the number of independent variables to get as simple an emergent space time structure of entropy and its generation as possible. One parameter being initial degrees of freedom, the second the minimum allowed grid size in space time, and the final parameter being emergent space time temperature. In order to initiate this inquiry, a comparison is made to two representations of a scale evolutionary Friedman equation, with one of the equations based upon LQG, and another involving an initial Hubble expansion parameter with initial temperature $T_{\text {Planck }} \sim 10^{19} \mathrm{GeV}$ used as an input into $T^{4}$ times $N(T)$. Initial assumptions as to the number of degrees of freedom have for $T_{\text {Planck }} \sim 10^{19} \mathrm{GeV}$ a maximum value of $N(T) \sim 10^{3}$. Making that upper end approximation for the value of permissible degrees of freedom is dependent upon a minimum grid size length as of about $l_{\text {Planck }} \sim 10^{-33}$ centimeters. Should the minimum uncertainty grid size for space time be higher than $l_{\text {Planck }} \sim 10^{-33}$ centimeters, then top value degrees of freedom of phase space as given by a value $N(T) \sim$ $10^{3}$ drops. In addition, the issue of bits, i.e. information is shown to not only have temperature dependence, but to be affected by minimum "grid size" as well.
\end{abstract}

\section{Keywords}

Entropy, Emergent Space Time, Normalized Energy Density 


\section{Introduction}

Recently, a big bounce has been proposed ${ }^{1}$ as an alternative to singularity conditions that Hawkings, Ellis [1], and others use. A quantum bounce, with a non zero but finite initial radius inevitably will lead to questions as to relic particle production, and of the amount of information bits surviving the big bounce, from a prior universe. This paper intends to find ways to configure scaling procedures to answer the question as to what would be optimal conditions for initial entropy production and byte of information "production" initially. To begin this inquiry we can start with examining candidates for the initial configuration of the normalized energy density. The normalized energy density of gravitational waves, as given by Maggiore [2] is

$$
\Omega_{g w} \equiv \frac{\rho_{g w}}{\rho_{c}} \equiv \int_{v=0}^{v=\infty} \mathrm{d}(\log v) \cdot \Omega_{g w}(v) \Rightarrow h_{0}^{2} \Omega_{g w}(v) \cong 3.6 \cdot\left[\frac{n_{v}}{10^{37}}\right] \cdot\left(\frac{v}{1 \mathrm{kHz}}\right)^{4}
$$

where $n_{v}$ is a frequency-based count of gravitons per unit cell of phase space. Equation (1.1) leads to, as given to Figure 1, candidates as to early universe models which should be investigated experimentally.

The author, Beckwith, wishes to determine inputs into $n_{v}$ above, in terms of frequency, and also initial temperature. Doing so will, if one gets inputs into Equation (1.1) right lead to examining how the arrow of time initial configuration, of entropy, influences choices as to models of what to chose from in terms of inflation. The author is convinced an answer to the above which will be dependent upon the number of degrees of freedom present in early universe cosmology. In

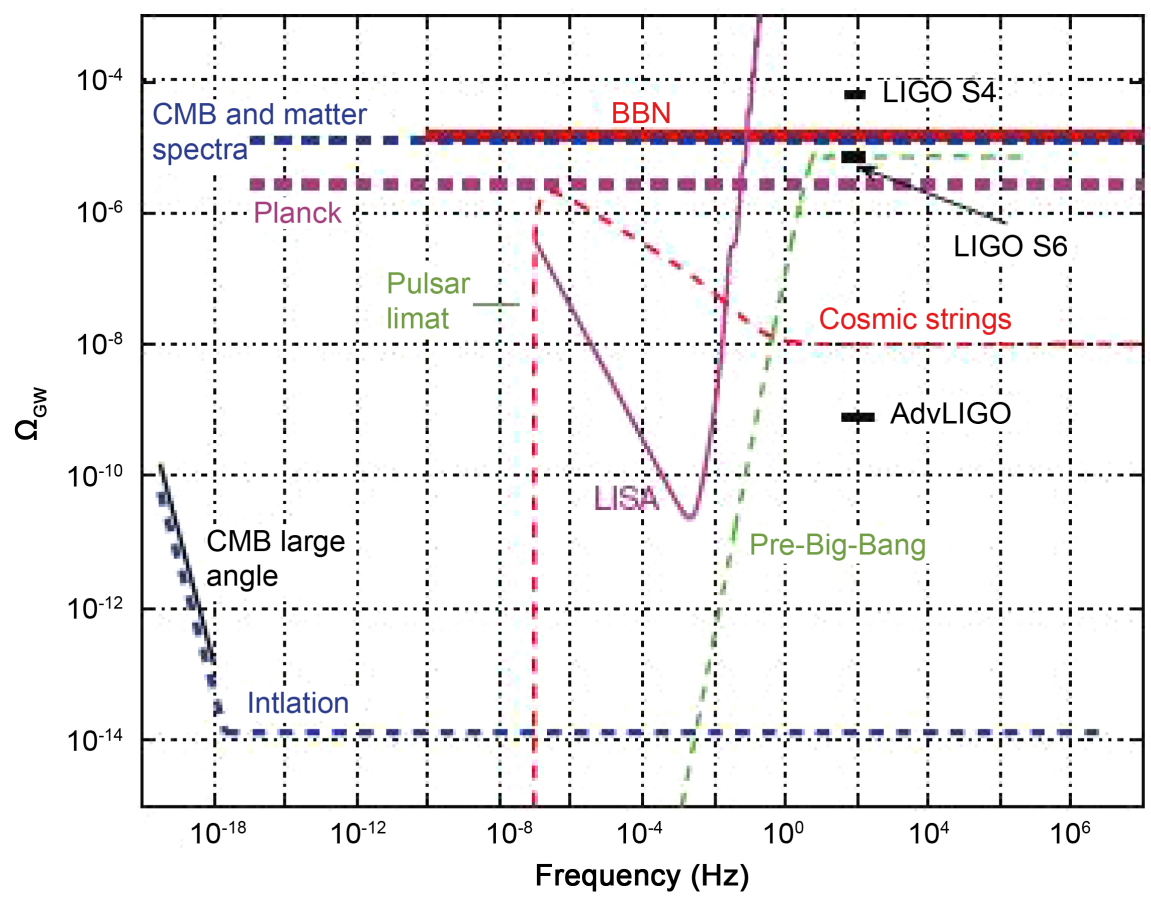

Figure 1. From Abbott et al. [4] (2009) shows the relation between $\Omega_{g}$ and frequency.

${ }^{1}$ Papers on LCQ at the 12th Marcell Grossman Meeting in 2009 (http://www.icra.it/MG/mg12/en/). 
the LQG version by [3], the Friedman equation may be written as follows: If conjugate momentum is in many cases, "almost" or actually a constant.

Doing so leads to, first considering, a non standard Friedman equation which is written up as [4]

$$
\left(\frac{\dot{a}}{a}\right)^{2} \equiv \frac{\kappa}{6} \cdot \frac{p_{\phi}^{2}}{a^{6}}
$$

This Equation (1.2) assumes that the conjugate dimension in this case has a quantum connection specified via an effective scalar field, $\phi$ obeying the relationship

$$
\dot{\phi}=-\frac{\hbar}{i} \cdot \frac{\partial}{\partial \cdot p_{\phi}}
$$

\section{How to Compare Equation (1.2) with Friedman Equation Behavior, If Thermal Influences Dominate Initially with $T_{\text {Planck }} \sim 10^{19} \mathrm{GeV}$}

This inquiry explicitly assumes a Friedman equation dominated by temperature with $N(T)$ a temperature dependent number of degrees of freedom present in a region of "phase space", and $\breve{a}$ a radiation constant, as given by Saunders (2005) [5]

$$
H^{2}=\left[4 \pi G \cdot \breve{a} \cdot T^{4} N(T) / 3 c^{2}\right] .
$$

If we make the following minimum uncertainty value for momentum as given by Baez-Olson [6], we have that $\Delta p \geq \hbar / l_{\text {Planck }}$ if $l_{\text {Planck }} \sim \Delta l$, i.e. what can we expect if there is a minimum value for the length of order Planck length, as opposed to the Ng and Van Damn [7] value of $\Delta p \geq \hbar / l^{1 / 3} l_{\text {Planck }}^{2 / 3}$, with $l \ll l_{\text {Planck }}$. Having made this choice of a minimum uncertainty grid, and if we set $\Delta p \approx \hbar / l_{\text {Planck }}=P_{\phi}$ and put that into Equation (1.2) one obtains the following to compare, as a way of obtaining $\mathrm{N}(\mathrm{T})$. Namely

$$
\left[4 \pi G \cdot \breve{a} \cdot T^{4} N(T) / 3 c^{2}\right] \approx \frac{\kappa}{6} \cdot \frac{\left[p_{\phi}^{2}=\left(\hbar / l_{\text {Planck }}\right)^{2}\right]}{a^{6}} .
$$

The consequence of Equation (1.5) would be to set conditions for which the following could be true.

$$
N(T) \sim 10^{3} \cong\left[\frac{c^{2} \kappa \cdot \hbar}{8 \pi G \cdot \breve{a}}\right] \cdot \frac{1}{T^{4} a_{\text {initial }}^{6} \cdot l_{\text {Planck }}^{2}} .
$$

If we take a dimensional re scaling of Equation (1.6), with

$$
N(T) \sim 10^{3} \sim \frac{1}{\left[T^{4} \approx T_{\text {Planck }}^{4}\right] \cdot\left[a_{\text {initial }}^{6}=10^{6 \cdot \beta}\right] \cdot l_{\text {Planck }}^{2}} .
$$

One can then obtain an algebraic equation to the effect that

$$
76-4 \delta^{+}-66+6 \beta \approx-3 \Rightarrow a_{\text {initial }} \sim 1 \text {. }
$$


This above approximation would be assuming that $T \sim 10^{19-\delta^{+}} \mathrm{GeV}$ i.e. close to the Planck temperature.

The other assumption is that the starting point for Planck expansion, has $a_{\text {initial }}=1$ with an enormous value for $a$ in the present era as opposed to another scaling convention that $a=[1 / 1+z]$ where one can have the red shift with values at the onset of inflation of the order of $z_{\text {initial }} \sim 10^{25}$ at the start of inflation, and $z_{\mathrm{CMBR}} \sim 1100-1000$ at the moment of CMBR photon radiation "turn on" with $z_{\text {Today }}=0$ in the present era. Examining what happens if one substitutes in for $l_{\text {Planck }} l^{1 / 3} l_{\text {Planck }}^{2 / 3}$ in Equation (1.7) would mean a substantially lower value for $N(T)$ if the following holds, i.e. $l \gg l_{\text {Planck }}$ making plausible even at the onset of inflation $N(T) \sim 10^{2}$ as reported by Kolb and Turner, 1991 [8], which is the usual value for degrees of freedom for the case of the electro weak era.

\section{First Principle Evaluation of Initial Bits of Information, as Opposed to Numerical Counting, and Entropy}

A consequence of Verlinde's [9] generalization of this technique as far as entropy, and the number of "bits" yields the following consideration, which will be put here for startling effect. Namely, if a net acceleration is such that $a_{\text {accel }}=2 \pi k_{B} c T / \hbar$ as mentioned by Verlinde [9] as an Unruth result, and that the number of "bits" is

$$
n_{\text {Bit }}=\frac{\Delta S}{\Delta x} \cdot \frac{c^{2}}{\pi \cdot k_{B}^{2} T} \approx \frac{3 \cdot(1.66)^{2} g^{*}}{\left[\Delta x \cong l_{p}\right]} \cdot \frac{c^{2} \cdot T^{2}}{\pi \cdot k_{B}^{2}} .
$$

This Equation (1.9) has a $T^{2}$ temperature dependence for information bits, as opposed to [10]

$$
S \sim 3 \cdot\left[1.66 \cdot \sqrt{\tilde{g}_{*}}\right]^{2} T^{3} .
$$

Should the $\Delta x \cong l_{p}$ order of magnitude minimum grid size hold, then conceivably when $T \sim 10^{19} \mathrm{GeV}$

$$
n_{\mathrm{Bit}} \approx \frac{3 \cdot(1.66)^{2} g^{*}}{\left[\Delta x \cong l_{p}\right]} \cdot \frac{c^{2} \cdot T^{2}}{\pi \cdot k_{B}^{2}} \sim 3 \cdot\left[1.66 \cdot \sqrt{\tilde{g}_{*}}\right]^{2} T^{3} .
$$

The situation for which one has [8] $\Delta x \cong l^{1 / 3} l_{\text {Planck }}^{2 / 3}$ with $l \gg l_{\text {Planck }}$ would correspond to having

$$
n_{\text {Bit }} \ll 3 \cdot\left[1.66 \cdot{\sqrt{\tilde{g}_{*}}}^{2}\right]^{2} T^{3}
$$

even if one has very high temperatures. Note that for WIMPS a situation as Y. J. $\mathrm{Ng}$ has it that [10] [11]

$$
S \cong n_{\text {Particle-Count }} .
$$

Note that Y. Jack Ng [10] [11] has $S \approx n_{\text {Particle-count }}$ for counting WIMP Dark Matter, with a much higher mass than what is observed with any accounting for 4 dimensional Gravitons. The current model WIMP model has individual par- 
ticles as of up to $100 \mathrm{GeV}$.

Next, if the additional degrees of freedom are warranted, comes the question of what are measurable protocols which may confirm/falsify this supposition. The following discussion will in part recap and extend a discussion which the author, Beckwith has presented in DICE 2010, in Italy [10].

\section{Consequences If There Are up to 1000 Degrees of Freedom, i.e. What If There Are Regimes of Space Time When Bits of Information Count Is Very Different from Particle Count for Entropy?}

The problem, though, is that there may be more than one graviton per information bit as given by Beckwith's calculations for entropy, and also energy carried per graviton. As given by Beckwith, in DICE 2010, Beckwith has made the following estimate, i.e. [10].

Note that J. Y. Ng uses the following [11], i.e. for DM, $S \sim n$, but this is for DM particles, presumably of the order of mass of a WIMP, i.e. $m_{\text {WIMP }} \approx 100 \mathrm{GeV} \sim 10^{11}$ electron volts, as opposed to a relic graviton mass-energy relationship [10]:

$m_{\text {graviton }}\left(\right.$ energy- $\left.v \approx 10^{10} \mathrm{~Hz}\right) \approx\left[100 \mathrm{GeV} \sim 10^{11} \mathrm{eV}-\right.$ WIMP $] \times 10^{-16} \sim 10^{-5} \mathrm{eV}$

If one drops the effective energy contribution to $v \approx 10^{0} \sim 1 \mathrm{~Hz}$, as has been suggested, then the relic graviton mass-energy relationship is:

$$
m_{\text {graviton }}\left(\text { energy- } v \approx 10^{0} \mathrm{~Hz}\right) \approx\left[100 \mathrm{GeV} \sim 10^{11} \mathrm{eV}-\mathrm{WIMP}\right] \times 10^{-26} \sim 10^{-15} \mathrm{eV} .
$$

Finally, if one is looking at the mass of a graviton a billion years ago, with

$$
m_{\text {graviton }}(\text { red-shift-value } \sim 0.55) \approx\left[100 \mathrm{GeV} \sim 10^{11} \mathrm{eV}-\mathrm{WIMP}\right] \times 10^{-38} \sim 10^{-27} \mathrm{eV}
$$

i.e. if one is looking at the mass of a graviton, in terms of its possible value as of a billion years ago, one gets the factor of needing to multiply by $10^{38}$ in order to obtain WIMP level energy-mass values, congruent with Y. Jack Ng's $S$ counting algorithm [10] [11]. What the author is suggesting, as he brought up in DICE 2010 is that the extra degrees of freedom may be necessary for obtaining clumps of $10^{38}$ gravitons to form coherent clumps to obtain GW of sufficient semi classical initial conditions, to obtain conditions, initially to have the $S \sim N$ counting algorithm work.

The author will later on attempt to prove that the $10^{38}$ factor so recorded is an artifact of Equation (1.9), i.e. that the scaling so implied in Equation (1.9) with the square of temperature, divided by grid size length means that for very light particles, the influence of high levels temperature will make the $10^{38}$ factor inevitable.

Still though, it would be important to come up with criteria as to how one can obtain a temperature and a mass of a "particle" regime for which $S \sim N$ work may be solvable via making the Ng. "Entropy" linkable to particle count. AND 
bits of information at the same time. To do so may entail introducing a new concept, that of "configurational entropy", as introduced below.

\section{Does $S_{C}$ as “Configurational Entropy" Serve as a Way to Make a One to One Connection between a Particle Count Algorithm of Entropy, and Bits of Information? No Matter What the "Mass" of a Particle and the Initial Background Temperature?}

The author has been advised that Rubi et al., 2008 [12] has a net temperature, as given by the following, namely for non equilibrium processes, one can look at $\tilde{T}$ as an effective temperature, and $S_{C}$ as "configurational entropy", and $e_{\text {Kin }}=m_{\text {graviton }} \cdot v_{\text {graviton }}^{2}$ as the kinetic energy of a graviton

$$
\frac{1}{\tilde{T}(x, v)}=\frac{\partial S_{C}}{\partial e_{K i n}} \text {. }
$$

In the case that the graviton has a very slight rest mass, one can, if [13] we pick $\mathrm{E}$ to be the rest energy, and $m_{\text {graviton }}$ the almost nonexistent rest mass of a graviton in four dimensions

$$
v_{\text {graviton }}^{2}=c^{2} \cdot\left[1-\left(m_{\text {graviton }}^{2} c^{4} / E^{2}\right)\right] .
$$

The net temperature may be considered to be a calculated function of a rise in temperature from almost nonexistent status, up to nearly Planck temperature, and the author is convinced, that one would have to, given different geometries, reconstruct the configurational entropy, once an idea of a minimum to the peak temperature, $T$, for Plank temperature values is obtained.

By doing so, the author hopes to obtain an evolution of $S_{C}$ with different values of the temperature, in order to come up with an emergent structure with $S_{C} \sim S \sim 3 \cdot\left[1.66 \cdot \sqrt{\tilde{g}_{*}}\right]^{2} T^{3}$. This should be done while paying attention to $\mathrm{t}^{\prime}$ Hooft's idea that an emergent structure would by necessity likely engage more than 100 dimensions, i.e. as Beckwith wrote about in [10], so how one defines Equation (1.17) may, with a proper definition of effective temperature, may force the adaptation of additional degrees of freedom.

\section{Conclusions. Extensions of This Thought Experiment, and Comparison with Entropy of Photons}

Recently, the author has been fortunate enough to obtain Leff's [14] entropy of photons per unit volume paper where for a phase space volume, $V$, and temperature $T$,

$$
S=(4 / 3) b V T^{3} \text {. }
$$

This should be compared with Beckwith's derived "graviton clumping" entropy result [10] per unit volume of phase space as given by $S \sim 3 \cdot\left[1.66 \cdot \sqrt{\tilde{g}_{*}}\right]^{2} T^{3}$ of Equation (1.10), and subsequent modifications as given in Equation (1.17).

What the author supposes, is that fine tuning the inter play between these two 
formulas, from the onset of inflation when there was likely coupling between gravitons, clumps of gravitons, and photons, may permit experimental measurements permitting investigation if there is an interplay between E \& $\mathrm{M}$ and gravity, and also modifications of gravity theory along the lines brought up by Sidharth [15], i.e. if Equation (1.10), Equation (1.17) and Equation (1.19) are representations of a joint phenomenon as is suggested by Sidarth's (which incidentally is for $\mathrm{E}$ and $\mathrm{M}$ radiation characterized by a given "carrier wave" frequency)

$$
A^{\mu}=\hbar \cdot \Gamma_{\mu}^{\mu v}
$$

where $A^{\mu}$ can be identified with the electromagnetic four potential. The idea, as Beckwith sees it would be to determine if there could be coupling between E \& $\mathrm{M}$ effects, and gravitation along the lines of employing the Quantum (coupled) oscillator frequency relationship for coherent "state" oscillation as given by Sidarth [15] via

$$
G \hbar \omega_{\max }=c^{5} .
$$

This would be to come up with a realistic way to talk about clumps of gravitons which may have coherent oscillatory behavior and to use this to make sense of the structure of up to $10^{38}$ coherent gravitons to form coherent clumps to obtain GW of sufficient semi classical initial conditions, to obtain conditions, initially to have the $S \sim N$ counting algorithm work for gravitons as coherent clumps, allegedly in a structure defined by Equation (1.21).

Then, after employing Equation (1.21) to next examine the limits of, and interexchange of effects given in Equation (1.14) and Equation (1.15) to determine from there to what degree is Equation (1.16) is giving us joint linkage of E\&M and gravitational waves in early universe conditions. Also, the author hopes that examining a potential inter play of Equation (1.10) to Equation (1.21) that the datum that the $10^{38}$ coherent gravitons [10] to form coherent clumps to obtain GW is necessary derivation will also, allow for explaining further the inter play between the choice of minimum length and momentum, as given by $\Delta p \approx \hbar / l_{\text {Planck }}=P_{\phi}$ and the supposition of more initial degrees of freedom than is usually supposed by conventional cosmology, of the sort presented by Kolb And Turner's book on cosmology. Finally, once this task is done, the author thinks that L. Glinka's formula [16] [17] of

$$
n_{f}=[1 / 4] \cdot\left[\sqrt{\frac{v\left(a_{\text {initial }}\right)}{v(a)}}-\sqrt{\frac{v(a)}{v\left(a_{\text {final }}\right)}}\right]
$$

could be investigated as being part of the bridge between phenomenology of both photon gases, and their entropy, as well as a modified treatment of L. Glinka's graviton gas [16] [17], with suitable inputs into the frequencies allowed for both "gases".

It is well worth noting that tests concerning the alleged Graviton gas should be tested against the predictions given in [18] by Dr. Corda, i.e. note this quote "it 
also showed some shortcomings and flaws which today advise theorists to ask if it is the definitive theory of gravity. In this essay we show that, if advanced projects on the detection of Gravitational Waves (GWs) will improve their sensitivity, allowing to perform a GWs astronomy, accurate angular and frequency dependent response functions of interferometers for $G W s$ arising from various Theories of Gravity, i.e. General Relativity and Extended Theories of Gravity, will be the definitive test for General Relativity".

Formula (1.22) as well would be useful in determining the would be existence of frequency response functions as far as a would be experimental datum for analysis, and also of falsifiable tests of alternatives to General Relativity as far as the foundation of gravity itself.

Finally, we should note as to the existence of positive identification of Gravitational waves. As seen in [19] and [20] there have been confirmation of GW,. Which afterwards is lending credibility to the would be frequency issue brought up in Equation (1.22) as well as testing of the criteria raised in [18] by Dr. Corda.

\section{Acknowledgements}

This work is supported in part by National Nature Science Foundation of China grant No. 11375279.

\section{References}

[1] Hawking, S. and Ellis, G.F.R. (1973) The Large Scale Structure of Space-Time. Cambridge University Press, Cambridge.

[2] Maggiore, M. (2008) Gravitational Waves, Volume 1: Theory and Experiment. Oxford University press, Oxford.

[3] Taveras, V. (2008) Corrections to the Friedmann Equations from LQG for a Universe with a Free Scalar Field. Physical Review D, 78, Article ID: 064072. http://arxiv.org/abs/0807.3325

[4] Abbott, B.P., et al. (2009) Gravity Ripples Chased. Nature, 460, 990-993.

[5] Sanders, R. (2005) Observational Cosmology. In: Papantonopoulous, E., Ed., The Physics of the Early Universe Lecture Notes in Physics, Volume 653, Springer-Verlag, New York, 105-137.

[6] Baez, J.C. and Olson, S.J. (2002) Uncertainty in Measurements of Distance. Classical and Quantum Gravity, 19, L121-L126. http://arXiv:gr-qc/0201030

[7] Ng, Y.J. and van Dam, H. (2000) Measuring the Foaminess of Space-Time with Gravity-Wave Interferometers. Foundations of Physics, 30, 795-805.

[8] Kolb, E. and Turner, S. (1994) The Early Universe. Westview Press, Chicago.

[9] Verlinde, E. (2010) On the Origin of Gravity and the Laws of Newton. http://arxiv.org/abs/1001.0785

[10] Beckwith, A.W. (2010) Gravitons Writ Large; i.e. Stability, Contributions to Early Arrow of Time, and Also Their Possible Role in Re Acceleration of the Universe 1 Billion Years Ago?"http://vixra.org/abs/1008.0060

[11] Ng, Y.J. and van Dam, H. (2000) On Wigner's Clock and the Detectability of Spacetime Foam with Gravitational-Wave Interferometers. Physics Letters B, 477, 429-435.

[12] Reguera, D. and Rubi, J.M. (2005) The Mesoscopic Dynamics of Thermodynamic 
Systems. http://arxiv.org/ftp/cond-mat/papers/0511/0511651.pdf

[13] Will, C. (2005) The Confrontation between General Relativity and Experiment. Living Reviews in Relativity, 9, 3. http://arxiv.org/pdf/gr-qc/0510072v2

[14] Leff, H. (2015) Fluctuations in Particle Number for a Photon Gas. American Journal of Physics, 83, 362. https://doi.org/10.1119/1.4904322

[15] Sidharth, B.G. (2010) Alternative Routes to Gravitation. Frontiers of Fundamental and Computational Physics: 10th International Symposium. AIP Conference Proceedings, 1246, 64-74. https://doi.org/10.1063/1.3460215

[16] Glinka, L.A. (2007) Quantum Information from Graviton-Matter Gas. Symmetry, Integrability and Geometry: Methods and Applications (SIGMA), 3, 87-100. https://doi.org/10.3842/SIGMA.2007.087

[17] Glinka, L.A. (2014) Thermodynamical Quantum Gravity. Applied Mathematics and Physics, 2, 66-72. https://doi.org/10.12691/amp-2-3-2

[18] Corda, C. (2009) Interferometric Detection of Gravitational Waves: The Definitive Test for General Relativity. International Journal of Modern Physics D, 18, 2275-2282. https://doi.org/10.1142/S0218271809015904

[19] Abbott, B.P., et al. (2016) Observation of Gravitational Waves from a Binary Black Hole Merger. Physical Review Letters, 116, 061102.

https://physics.aps.org/featured-article-pdf/10.1103/PhysRevLett.116.061102

[20] Abbott, B.P., et al. (2016) GW151226: Observation of Gravitational Waves from a 22-Solar-Mass Binary Black Hole Coalescence. Physical Review Letters, 116, 241103. https://doi.org/10.1103/PhysRevLett.116.241103 